Pure Appl. Chem., Vol. 75, No. 8, pp. 1041-1047, 2003.

(C) 2003 IUPAC

\title{
Triplet carbenes: From fleeting existence to attractive molecular units*
}

\author{
Hideo Tomioka \\ Chemistry Department for Materials, Faculty of Engineering, Mie University, Tsu, \\ Mie 514-8507, Japan
}

Abstract: Attempts to stabilize the triplet state of carbenes to the extent that they can survive under normal conditions were made, in which triplet carbenes having a half-life of $19 \mathrm{~min}$ are realized. A way to use the resulting persistent carbene as a unit to construct photosensitive magnetic materials is also discussed.

\section{INTRODUCTION}

Many organic photoreactions proceed by multistep processes in which intermediate molecular species are involved in the conversion from one molecule to another. Since organic chemistry is basically the chemistry of carbon compounds, it is natural that the greatest attention has been paid to reactive intermediates that contain carbon. The normal state of carbon is tetravalent. The most common reactive intermediates formed in the conversion of one tetravalent-carbon compound into another incorporated trivalent carbon, e.g., a carbanion (1), a free radical (2), and a carbocation (3). The chemistry of these trivalent species has been broadly worked out over the past 70 years or so and is relatively thoroughly understood.

A normal carbon atom can be manipulated to an even greater degree by removing two of the atoms ordinarily bonded to it. This results in the formation of the enormously reactive divalent species known as carbenes (4) [1]. The history of carbenes is much shorter, and their chemistry is understood less well than that of the trivalent species. Moreover, their great instability and inherently complex nature have made them far more exotic than the trivalent species.

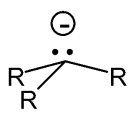

1

Carbanion<smiles>[R]C([R])C</smiles>

2

Radical<smiles>[R]C([R])[R]</smiles>

3

Carbocation

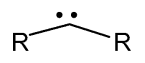

4

Carbene

Much of the interest in carbene derivatives originates from the relationship between their electronic nature and molecular structures. Carbenes (4) have two nonbonding (or free) electrons and, therefore, can have two electronic states depending on the direction of spin of the electrons. They can exist as a singlet state $\left({ }^{1} \mathbf{4}\right)$ that has antiparallel spins and as a triplet state $\left({ }^{3} \mathbf{4}\right)$ that has parallel spins.

* Lecture presented at the XIX ${ }^{\text {th }}$ IUPAC Symposium on Photochemistry, Budapest, Hungary, 14-19 July 2002. Other presentations are published in this issue, pp. 999-1090. 


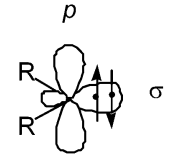

Singlet Carbene $\left({ }^{1} 4\right)$

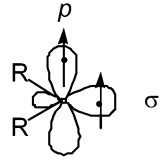

Triplet Carbene $\left({ }^{3} \mathbf{4}\right)$

Generally speaking, singlet states show extremely high reactivity and are capable of reacting even with very inert sites, such as $\mathrm{C}-\mathrm{H}$ bonds. Triplet states, even though less useful as reactive intermediates because of their sluggishness, attract much attention as a spin source. Moreover, carbenes can be easily and cleanly generated from the corresponding diazo compounds (5) upon photoexcitation, which makes carbenes very attractive reagents in photosensitive materials. Thus, singlet carbenes play a key role in photosensitive materials, such as photoresists and photoaffinity labels, while their triplet counterparts have attracted much attention as a potential spin source for photoresponsive magnetic materials.

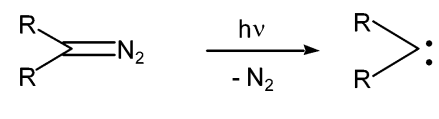

The present work was undertaken (1) to stabilize the triplet state of carbenes to the extent that they can survive under normal conditions and (2) to use the resulting persistent carbene as a unit to construct photosensitive magnetic materials.

\section{PERSISTENT TRIPLET CARBENE}

There are two ways to stabilize reactive intermediates, either thermodynamically or kinetically. More specifically, thermodynamic stabilization is the way to decrease the potential energy of the species in question. In organic molecules, this is usually achieved electronically by the resonance effect. This effect is shown to be very effective for singlet carbenes because the singlet has a vacant $\pi$ orbital, and, hence, it is relatively easily stabilized by accepting nonbonding electron pairs from adjacent heteroatom substituents. For instance, the phosphinocarbene is isolated as a stable crystal with a melting point above $100{ }^{\circ} \mathrm{C}$. However, in this case, the contribution of phosphinoalkyne is very large, and, hence, the interpretation of these species as "free" carbenes has been a topic of debate, since the contribution of the ylidic nature as a result of $\mathrm{p} \pi$-p $\pi$ delocalization plays a crucial role in these carbenes.

Kinetic stabilization, on the other hand, is the way to increase the activation energy of the decomposition pathway of the species while keeping the potential energy essentially constant. This is usually done by protecting the reactive center from external reagents by introducing bulky but unreactive substituents around the reactive center, which is often called steric protection. Triplet carbenes are less prone to thermodynamic stabilization because they do not have suitable orbitals, and, therefore, the triplet state is more effectively stabilized by steric protection. This method has an advantage over the thermodynamic one, as it keeps electronic properties essentially intact. Therefore, we have attempted to stabilize triplet diphenylcarbenes (DPCs) by introducing various substituents at the ortho position as kinetic protectors.

\section{Triplet diphenylcarbenes}

The lifetime of the triplet DPCs was greatly increased as chlorine (6), methyl (7), and bromine substituents were introduced at the ortho positions, and bis(2,6-dibrormo-4-tert-butylphenyl)carbene (8) 
was found to have a half-life of approximately $20 \mathrm{~s}$ in degassed benzene at room temperature [2]. A carbene surviving for more than a few seconds can be regarded as very stable for a triplet carbene, but this lifetime is still ephemeral for a "real" molecule. Therefore, we need to explore a kinetic protector for a triplet carbene that is bigger than the bromine atom and yet unreactive toward a triplet carbene. The $\mathrm{CF}_{3}$ group is a very promising kinetic protector for carbenes, since it is much bigger than the bromine atom, and, more importantly, $\mathrm{C}-\mathrm{F}$ bonds are known to be the only type of bond unreactive toward a carbene center.<smiles>Clc1cc(Cl)c(Cc2c(Cl)cc(Cl)cc2Cl)c(Cl)c1</smiles>

6

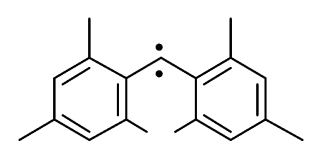<smiles></smiles>

8

However, it is extremely difficult to prepare a precursor diphenyldiazomethane that has four $\mathrm{CF}_{3}$ groups at all of the ortho positions. Therefore, we decided to use other sterically less demanding groups in combination with the $\mathrm{CF}_{3}$ group.

The diphenyldiazomethane that we managed to prepare was the one (9) with two bromine substituents in addition to two $\mathrm{CF}_{3}$ groups on the other phenyl ring. The photolysis of 9 generated the transient absorption bands ascribable to the triplet carbene ${ }^{3} \mathbf{1 0}$. The decay of the absorption as a function of time indicated that it took more than $1 \mathrm{~h}$ before all the absorption disappeared completely under these conditions. The curve was analyzed in terms of second-order decay, and the lifetime was estimated in the form of half-life to be $10 \mathrm{~min}$. Thus, a triplet carbene with a lifetime over several minutes was realized for the first time [3].<smiles>CC(C)(C)c1cc(F)c(C(=N)c2c(Br)cc(C(C)(C)C)cc2C(F)(F)F)c(Br)c1</smiles>

9

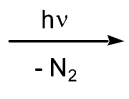<smiles></smiles>

10

\section{Triplet di(9-anthryl)carbene}

Among the many triplet diarylcarbenes known, the triplet di(9-anthryl)carbene (12) is unique, as it shows the smallest $D\left(0.113 \mathrm{~cm}^{-1}\right)$ and $E\left(0.0011 \mathrm{~cm}^{-1}\right)$ values ever reported [4]. This means that the carbene has an almost linear and perpendicular geometry with extensive delocalization at the unpaired electrons into the anthryl portions of the molecule. The extensive delocalization is expected to stabilize this carbene thermodynamically, while the perpendicular geometry of the anthryl group stabilizes the carbene center kinetically through shielding with the four peri-hydrogens. That is, the electronic factor and the molecular structure of the molecule seem ideal for the formation of a stable triplet carbene.

In spite of those highly favorable structural factors, ${ }^{3} \mathbf{1 2}$ is very ephemeral: its lifetime in degassed benzene is $0.5 \mu \mathrm{s}$, shorter than that of even the parent triplet DPC. Product analysis studies have shown that ${ }^{3} 12$ forms a trimer (13) of dianthrylcarbene as the main product $(50-60 \%)$ [5]. The structure of the trimer (13) is characterized as one formed as a result of a three-fold coupling at position 10 of the carbene in a cyclic fashion. This observation suggests that delocalization of the unpaired electrons in ${ }^{3} \mathbf{1 2}$ leads to their leaking out from the carbene center to position 10, where sufficient spin density builds up for the trimerization to take place.

The above observations indicate that the stability of ${ }^{3} \mathbf{1 2}$ would increase if the trimerization reaction were somehow suppressed. Thus, we attempted to generate a dianthrylcarbene with a substituent at position 10 to block the reactivity there. Usually, the tert-butyl group is the best substituent of such 
<smiles></smiles>

11

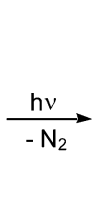<smiles></smiles>

12

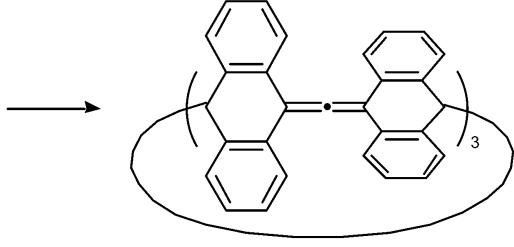

13

a blocking group, but we found that the tert-butyl group at position 10 of the anthryl ring is very sensitive to an acid and easily removed during the procedure employed to prepare the precursor diazo compound. Thus, we introduced the phenyl group as a blocker, which indeed turned out to be very effective.

A triplet carbene generated by the photolysis of bis[9-(10-phenyl)anthryl]diazomethane (14) in 2-methyltetrahydrofuran (MTHF) glass at $77 \mathrm{~K}$ gave electron spin resonance (ESR) signals that were very similar to those observed for dianthrylcarbene. The zero-field splitting (zfs) parameters $(D=0.105$ $\mathrm{cm}^{-1}, E=4.4 \times 10^{-4} \mathrm{~cm}^{-1}$ ) derived from the signals are essentially the same as those obtained for ${ }^{3} \mathbf{1 2}$. This indicates that the phenyl groups are not in the same plane as the anthryl rings owing to the repulsion between ortho- and peri-hydrogens. The ESR signals of ${ }^{3} 12$ and ${ }^{3} 15$ differ only in their thermal stability. When the MTHF glass containing ${ }^{3} \mathbf{1 2}$ was gradually warmed, the signals due to ${ }^{3} \mathbf{1 2}$ started to disappear at around $90 \mathrm{~K}$, whereas no significant decay of the signals of ${ }^{3} 15$ was observed up to $240 \mathrm{~K}$. The signal of ${ }^{3} \mathbf{1 5}$ started to decay only at around $270 \mathrm{~K}\left(\sim 0{ }^{\circ} \mathrm{C}\right)$ but was still visible when heating to $300 \mathrm{~K}\left(\sim 27^{\circ} \mathrm{C}\right)$.

To prove the stability of ${ }^{3} \mathbf{1 5}$ under normal conditions, 14 was photolyzed in degassed benzene at room temperature. The bands due to ${ }^{3} \mathbf{1 5}$ decayed cleanly, showing isosbestic points, and very slowly, persisting for more than $3 \mathrm{~h}$ before disappearing completely. The decay curve was analyzed in terms of second-order kinetics $\left(2 \mathrm{k} / \varepsilon l=5.2 \times 10^{-4} \mathrm{~s}^{-1}\right)$. The half-life for ${ }^{3} \mathbf{1 5}$ was estimated to be $19 \mathrm{~min}$. This is the longest-lived triplet carbene thus far generated in our research group [6].<smiles></smiles>

\section{TOWARD PERSISTENT HIGH-SPIN POLYCARBENES}

Triplet carbenes are regarded as one of the most effective spin sources [7]. This is because the magnitude of the exchange coupling between the neighboring centers is large and the photolytic production of polycarbenes is possible even in solid media at cryogenic temperatures under helium. Furthermore, in situ magnetic measurements are possible if poly(diazo) precursors are available. Indeed, Iwamura and his coworkers prepared a "starburst"-type nonadiazo compound (16) and demonstrated that the nine diazo groups are photolyzed from them at low temperature to give an $\mathrm{S}=9$ ground-state polycarbene (17) [8]. However, those systems lack stability for characterization under ambient conditions and have an inherent drawback for further extension to usable magnetic materials. Moreover, the synthesis of the precursor diazo compounds becomes more difficult and laborious as the number of carbene units increases.

In order to overcome these difficulties in generation of usable macroscopic spins, a method to stabilize triplet carbene centers and then connect them in a ferromagnetic fashion has to be developed.

As we have fairly stable triplet carbenes, the next step should be to explore a way to connect them while retaining a robust $\pi$-spin polarization. The topology-mediated molecular design of neutral organic 


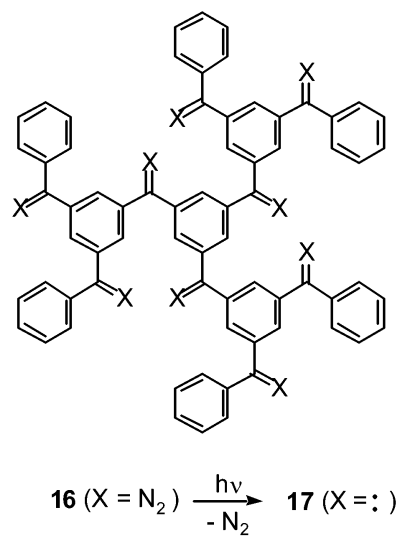

high-spin systems of $\pi$-conjugation has been well established in the field of purely organic magnetics. In order to use polybrominated triplet DPCs as persistent spin units and construct as high a spin as possible, we designed 1,3,5-tris(ethynyl)phenyl as a two-dimensional linker.

The reaction scheme for the coupling of the persistent carbene precursor $\mathbf{2 0}$ is summarized in Scheme 1 [9]. Diazo groups are generally labile, and therefore they are usually introduced at the last step of the synthesis when preparing both mono(diazo) and poly(diazo) compounds. Application of this strategy to these highly sterically congested diazo compounds, prepared usually by the base treatment of $N$-nitrosocarbamates, is almost impossible. Fortunately, polybromodiphenyldiazomethanes $\mathbf{1 8}$ and 19 were found to be stable enough to survive in the presence of $\operatorname{Pd}(0)$ and $\mathrm{Cu}(\mathrm{l})$ under Sonogashira coupling reaction conditions. This is obviously due to the fact that the four bromine atoms at the ortho positions, which effectively protect the carbene center from the external reagents, are also able to protect the diazo carbon equally well. Moreover, each of the four ortho carbons is also protected from the approach of external reagents by these bromine substituents. This enabled us to introduce connecting groups at the para-position of the monomer diazo compound (e.g., 18) to give the functionalized monomer 19 and then connect it to an appropriate linker that satisfies the topological requirement for

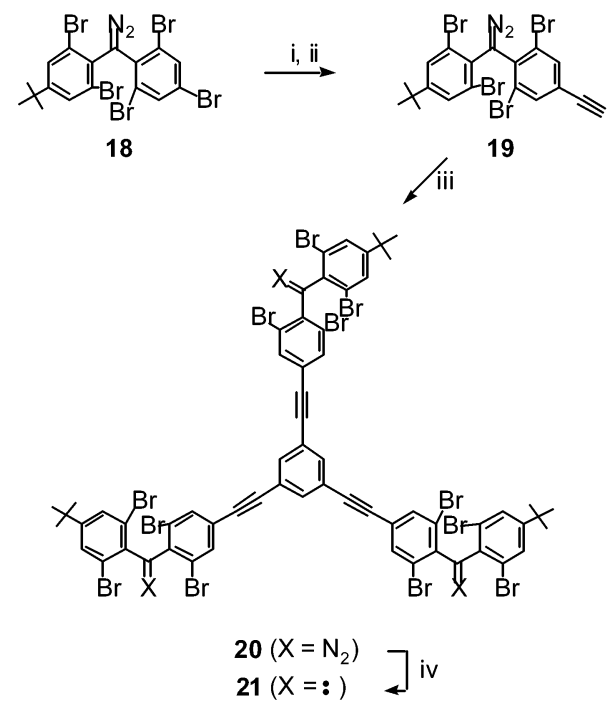

Scheme 1 (i) $\mathrm{Me}_{3} \mathrm{SiC} \equiv \mathrm{CH} /\left(\mathrm{Ph}_{3} \mathrm{Ph}\right)_{2} \mathrm{PdCl}_{2} / \mathrm{Cul}$; (ii) $\mathrm{KOH} / \mathrm{MeOH}$; (iii) $1,3,5-\mathrm{I}_{3} \mathrm{C}_{6} \mathrm{H}_{3} /\left(\mathrm{Ph}_{3} \mathrm{P}\right)_{2} \mathrm{PdCl}_{2} / \mathrm{Cul}$; (iv) hv $(\lambda>300 \mathrm{~nm})$. 
intramolecular ferromagnetic spin alignment. Thus, we were able to introduce an ethynyl functional group very selectively at the para-position in a reasonable yield. Deprotection of the trimethylsilyl group proceeded almost quantitatively to give 19. Subsequent coupling with 1,3,5-triiodobenzene took place smoothly to produce tris(diazo) compound 20, where three carbene precursor units were properly introduced so as to generate tris(carbene) (21) connected in a ferromagnetic fashion

Photolysis of $\mathbf{2 0}$ in an MTHF glass at $5 \mathrm{~K}$ gave a fine-structure ESR spectrum, which was different from that observed by the photolysis of the corresponding mono-diazomethane. The salient features of organic high-spin species apparently failed to appear in the observed spectrum. Canonical peaks dominated in the $g \sim 2$ range, and the key peaks appearing in the wings away from the $g \sim 2$ region were only vague. It is obvious that conventional continuous-wave (CW) ESR spectroscopy fails to give evidence of the spin identification of the tris(carbene).

In order to identify the spin multiplicity of the tris(carbene), field-swept two-dimensional electron spin transient nutation (2D-ESTN) spectroscopy [10] was utilized. This technique is based on a pulsed FT ESR spectroscopic method and is capable of providing straightforward information on electronic and environmental structures of high-spin species even in amorphous materials, something that conventional CW ESR cannot accomplish. The nutation spectra unequivocally demonstrated that the observed fine-structure spectrum is due to a spin-septet state.

A nutation peak arising from any doublet species of by-products was not detected, showing the remarkable chemical stability of the tris(carbene) $\mathbf{2 1}$. Furthermore, the marked thermal stability of $\mathbf{2 1}$ manifested itself during the annealing process. A significant change in the ESR signal shape was observed when the matrix temperature was raised above $140 \mathrm{~K}$ and maintained for $15 \mathrm{~min}$. New fine-structure signals were irreversible with the temperature and characteristic of small fine-structure constants. Using 2D-ESTN spectroscopy, it was also shown that they originate in a septet ground state. This is in sharp contrast with what was observed for septet-state benzene-1,3,5-tris(phenylmethylene), which has been shown to be persistent only up to 85 and $50 \mathrm{~K}$ in rigid glasses.

These studies indicate that the diazo compounds prepared as precursors for persistent triplet carbenes are also very stable and survive during further chemical functionalization so that they can serve as building blocks for persistent high-spin polycarbenes. These building blocks are very useful, since they can be attached to properly designed $\pi$-topological linkers essentially ad libitum. Moreover, the undesirable bimolecular side reactions that generate chemical defects among the topologically controlled high-spin $(S=1)$ centers for extended spin alignment are rigorously avoided. These findings open a new door to the design and preparation of new persistent high-spin molecules for more practical organic ferromagnetics.

\section{ACKNOWLEDGMENT}

The authors are grateful to the Ministry of Education, Science and Culture of Japan for support of this work through a Grant-in-Aid for Scientific Research for Specially Promoted Research (No. 12002007).

\section{REFERENCES}

1. For reviews of general reactions of carbenes, see (a) W. Kirmse. Carbene Chemistry, $2^{\text {nd }}$ ed. Academic Press, New York (1971); (b) Carbenes, Vols. 1 and 2, R. A. Moss and M. Jones, Jr. (Eds.), Wiley, New York (1973 and 1975); (c) Carbene(oide), Carbine, M. Regitz (Ed.), Thieme, Stuttgart (1989).

2. (a) H. Tomioka. Acc. Chem. Res. 30, 315 (1997); (b) H. Tomioka. In Advances in Carbene Chemistry, U. Brinker (Ed.), Vol. 2, pp. 175-214, JAI Press, Greenwich, CT (1998); (c) H. Tomioka. In Advances in Strained and Interesting Organic Molecules, B. Halton (Ed.), Vol. 8, pp. 83-112, JAI Press, Greenwich, CT (2000); (d) H. Tomioka. In Carbene Chemistry, G. Bertrand (Ed.), pp. 103-152, Fontis Media S. A., Lausanne (2002). 
3. K. Hirai and H. Tomioka. J. Am. Chem. Soc. 121, 10213 (1999).

4. (a) E. Wasserman, V. J. Kuck, W. A. Yager, R. S. Hutton, F. D. Greene, V. P. Abegg, N. M. Weinshenker. J. Am. Chem. Soc. 93, 6335 (1971); (b) D. J. Astles, M. Girard, D. Griller, J. Kolt, D. D. Wayner. J. Org. Chem. 53, 6053 (1998).

5. Y. Takahashi M. Tomura, K. Yoshida, S. Murata, H. Tomioka. Angew. Chem., Int. Ed. 39, 3478 (2000).

6. H. Tomioka, E. Iwamoto, H. Itakura, K. Hirai. Nature 412, 6847 (2001).

7. H. Iwamura. Adv. Phys. Org. Chem. 26, 179 (1990).

8. K. Matsuda, N. Nakamura, K. Inoue, N. Koga, H. Iwamura. Bull. Chem. Soc. Jpn. 69, 1483 (1996).

9. H. Tomioka, M. Hattori, K. Hirai, K. Sato, D. Shiomi, T. Takui, K. Itoh. J. Am. Chem. Soc. 120, 1106 (1998).

10. T. Takui, K. Sato, D. Shiomi, K. Itoh, T. Kaneko, E. Tsuchida, H. Nishide. In Magnetism; A Supramolecular Function, O. Kahn (Ed.), pp. 249-280, Kluwer, Dordrecht (1996). 